\title{
Investigación y movilidad internacional: análisis de las estancias en centros extranjeros de los investigadores andaluces
}

\author{
Carolina Cañibano*, Javier Otamendi ${ }^{\star \star}$ y Francisco Solís ${ }^{\star \star \star}$
}

Resumen: La movilidad temporal internacional de los investigadores es un importante mecanismo de difusión de conocimiento y emergencia y desarrollo de redes de colaboración científica. El conocimiento empírico de este tipo de flujos geográficos es, sin embargo, muy limitado, debido a una generalizada escasez de datos en la mayoría de los países. En este trabajo recurrimos a la información de los currículos contenidos en el Sistema de Información Científica de Andalucía para analizar las dinámicas de movilidad temporal internacional de los investigadores andaluces. Estudiamos a una población de más de 6.000 investigadores móviles y casi 18.000 estancias realizadas entre 1961 y 2009. El análisis revela significativas tendencias de cambio a lo largo del tiempo en la frecuencia, duración y destino de las estancias, así como en las diferencias por género. Asimismo, pone de manifiesto marcadas diferencias en los perfiles de movilidad por área de conocimiento. El trabajo constituye una contribución metodológica en la medida en que emplea por primera vez datos curriculares electrónicos para el estudio del fenómeno de la movilidad y de este tipo de tendencias.

Palabras clave: movilidad de investigadores, curriculum vitae, sistema de información curricular, estancias internacionales de investigación

\section{Research and mobility: analysis of international research visits of Andalusian researchers}

Abstract: The temporary international mobility of researchers is a key mechanism for the dissemination of scientific knowledge and the creation and development of scholarly collaborative networks. However, empirical knowledge about international research visits is very limited due to an overall lack of data in most countries. In this paper we use curriculum vitae data contained in the Scientific Information System of Andalusia to study the dynamics of the temporary mobility of Andalusian researchers. We analyse a population of more than 6,000 mobile researchers and almost 18,000 international research visits, over the period 1961-2009. The analysis reveals significant changes over time in the frequency, duration, geographical destination and gender distribution of visits. It also shows important differences in temporary mobility patterns among research

* INGENIO (Universidad Politécnica de Valencia-CSIC) y Universidad Rey Juan Carlos. Correo-e: ccanibano@ingenio.upv.es.

** Universidad Rey Juan Carlos. Madrid. Correo-e: franciscojavier.otamendi@urjc.es.

*** Consejería de Economía, Innovación y Ciencia; Junta de Andalucía. Sevilla. Correo-e: franciscom.solis@juntadeandalucia.es.

Recibido: 2-07-09; 2. ${ }^{\text {a }}$ versión: 28-01-10; aceptado: 23-02-10. 
disciplines. The study makes a methodological contribution to the literature, as it applies electronic curriculum data to the analysis of trends in this type of mobility for the first time.

Key Words: mobility of researchers, curriculum vitae, curricular information system, international research visits.

\section{Introducción}

La creación y difusión de conocimiento científico han estado asociadas a la movilidad geográfica de los investigadores desde el inicio de la historia escrita. Atenas fue el mayor foco de atracción de intelectuales en el Mundo Antiguo hasta aproximadamente 300 a. C. (US House of Representatives, 1977). La migración de académicos y estudiantes se convirtió en un fenómeno habitual en la Edad Media (Brandi, 2006) y ha seguido siéndolo desde entonces. El fenómeno empezó a atraer la atención de investigadores sociales y de los políticos a raíz de las migraciones masivas que se produjeron desde Europa hacia Norteamérica en los años posteriores a la Segunda Guerra Mundial y, posteriormente, a raíz del incremento en los flujos de personal científico y técnico procedente de Asia, Europa del Este y algunos países emergentes hacia países con sistemas científicos más consolidados. Algunos de estos movimientos han sido de "ida y vuelta" (Saxenian, 2006), como el caso de los tecnólogos chinos e indios que iniciaron sus carreras en Silicon Valley antes de regresar a sus países de origen. Otros flujos de movilidad internacional han originado la organización de diásporas científicas de investigadores de determinadas nacionalidades (Meyer, 2001). Asimismo, determinados flujos de movilidad han constituido la base para el establecimiento de vínculos de cooperación y co-producción (De Filippo y otros, 2007; Fontes, 2007; Jonkers y Tijssen, 2008).

En el marco de la Estrategia Europea de Lisboa, que aspira a transformar la Unión Europea en una economía basada en el conocimiento capaz de generar altos niveles de empleo y crecimiento económico (Consejo Europeo de Lisboa, 2000), la movilidad de los investigadores se erige como un fenómeno clave y estratégico para la integración del Espacio Europeo de Investigación (CEC, 2001). La Unión Europea quiere asentar una quinta libertad que se sume a las cuatro ya operativas (capitales, bienes, servicios y personas): la libertad de circulación del conocimiento y la tecnología (CEC, 2007). Las características del conocimiento científico hacen que la difusión internacional del mismo requiera, en muchas ocasiones, el contacto personal entre los investigadores, lo que sólo es posible si éstos se trasladan de un país a otro.

Por lo general, existe consenso acerca de la importancia de la movilidad de los investigadores, lo que queda reflejado en el peso que el fenómeno ha ido adquiriendo en las políticas públicas de investigación. No es menos cierto, sin embargo, que sus características permanecen fundamentalmente desconocidas para los responsables del diseño de estas políticas. ¿Con qué frecuencia se des- 
plazan los investigadores a otros países? ¿con qué objetivos? ¿qué proporción de esos movimientos son de ida y vuelta? ¿qué países resultan más atractivos para los investigadores? ¿qué efectos tiene la movilidad? ¿cómo difieren los patrones de movilidad entre áreas de conocimiento? Todas éstas son preguntas relevantes desde el punto de vista del diseño de las políticas públicas y que diversos investigadores hemos intentado abordar, especialmente a lo largo de la última década. El obstáculo fundamental para la observación y comprensión empírica del fenómeno ha sido, hasta la fecha, la escasez de datos.

En este artículo recurrimos a una nueva fuente de información con enorme potencial, para analizar las dinámicas de movilidad temporal de los investigadores andaluces. Se trata de la información curricular contenida en el Sistema de Información Científica de Andalucía. Las características de este sistema y de la información descargada del mismo, nos permiten estudiar los patrones de movilidad temporal de toda la población de investigadores andaluces móviles (más de 6.000), lo cual supone un importante salto metodológico en comparación con los estudios disponibles anteriores, tanto en nuestro país como en otros. El trabajo revela el potencial que el sistema de información curricular nacional que está en desarrollo en España puede llegar a tener para la evaluación y seguimiento de la actividad de los recursos humanos del sistema científico.

El análisis realizado aporta nuevo conocimiento acerca de los patrones de movilidad temporal de la población estudiada, en función del género, la edad, el área de conocimiento, los países de destino y la duración y frecuencia de las estancias registradas. Los resultados apuntan que los patrones de movilidad han cambiado a lo largo del tiempo y que difieren notablemente entre disciplinas científicas.

El artículo se estructura de la forma siguiente: a modo de introducción conceptual, repasamos en primer lugar la literatura más relevante disponible, relativa a la movilidad de los investigadores en general y a la movilidad temporal en particular. Seguidamente presentamos la metodología del análisis curricular y los recientes desarrollos en este campo, para pasar a describir brevemente el sistema de información curricular que está en desarrollo en España y las características del Sistema de Información Científica de Andalucía, del que se han obtenido los datos para el estudio. Finalmente, a modo de conclusiones, presentamos y comentamos los resultados del análisis.

\section{Movilidad temporal e investigación científica}

La actividad científica e investigadora es de suyo "móvil» e itinerante, especialmente en su dimensión geográfica. Gaillard y Gaillard (1997) identifican la movilidad como un fenómeno socio-antropológico, característico del quehacer científico. Por su parte, Meyer y otros (2001), califican de "nuevo nomadismo científico" a la tendencia al incremento de flujos migratorios internacionales, "multilaterales y policéntricos" que se viene observando desde la década de 1990.

Rev. Esp. Doc. Cient., 33, 3, julio-septiembre, 428-457, 2010. ISSN: 0210-0614. doi:10.3989/redc.2010.3.736 
Movilidad y migración son no obstante dos fenómenos distintos, por más que el primero de ellos englobe al segundo. La movilidad es movimiento de un espacio a otro. Puede darse entre países, regiones, organizaciones, sectores e incluso entre disciplinas científicas, lo cual ha sido calificado como "movilidad intelectual" en el ámbito de la sociología de la ciencia (Shinn y Benguigui, 1997). En este último caso, el investigador se estaría moviendo de un espacio mental a otro. El fomento de todas estas movilidades, incluida la intelectual, se ha convertido en uno de los ejes centrales de la política europea de recursos humanos para la ciencia y la tecnología (CEC, 2001, 2007, 2008a). También es una prioridad de la política científica española (Plan Nacional: Programa Nacional de Movilidad de Recursos Humanos ${ }^{1}$ ), así como de la andaluza (Plan Andaluz de Investigación, Desarrollo e Innovación. Línea estratégica: capital humano y social).

Por lo general, existe consenso acerca de los beneficios de la movilidad. La movilidad confronta al individuo con culturas y situaciones distintas, lo que frecuentemente favorece la emergencia de nuevas ideas y descubrimientos (Johnson, 1968); favorece la transmisión del conocimiento que no puede circular por otras vías, por estar estrechamente asociado a determinados contextos o a determinadas personas y fomenta la emergencia y el desarrollo de redes de colaboración, (re)configurando el capital social que vincula a individuos y colectivos (Bozeman y otros 2001). Esta creencia generalizada en los beneficios de la movilidad se ve acompañada, en muchos casos, por el miedo a la descapitalización intelectual o a la "fuga de cerebros" asociada a la emigración de los investigadores, la cual se asume afecta negativamente a los organismos, países, sectores e incluso disciplinas que el investigador abandona al moverse (Bhagwati, 1979; Kapur y McHale, 2005). Hemos reflexionado en otro trabajo acerca de la consistencia y fundamentos teóricos de estas ya arraigadas convicciones, aparentemente opuestas (Cañibano, 2008).

La tipología de la movilidad atiende también a su duración. Puede tratarse de una movilidad de duración indeterminada o claramente temporal. La mayor parte de los trabajos que tratan la movilidad de los investigadores y su migración como fenómenos equivalentes centran su atención, únicamente, en un tipo de movilidad entre todos los posibles: la geográfica, internacional y con vocación de permanencia. Al ser ésta motivo de preocupación para los países de origen, especialmente a partir de la década de 1960, es la que ha recibido más atención por parte de los investigadores sociales y los gobiernos. Dentro de la generalizada dificultad de observación empírica del fenómeno, se trata además de un tipo de movilidad más fácilmente observable y medible, a través de las estadísticas de migración y los censos de población. No es menor, no obstante, la dificultad que implica el hecho de que la profesión de "investigador" no esté identificada en las clasificaciones habituales de ocupaciones. No lo está en la Clasificación Internacional de Ocupaciones de la OCDE. Las estimaciones acerca de los flujos migratorios de investigadores han de guiarse, normalmente, por el

\footnotetext{
${ }^{1}$ http://www.planacionalidi.es.
} 
nivel educativo de los migrantes o han de basarse en encuestas específicas. No obstante, la importancia de los flujos de movilidad temporal ha sido destacada también, especialmente en la medida en que van en aumento debido al abaratamiento y facilidad del transporte y a la formación de redes internacionales de colaboración cada vez más extensas (CEC, 2001; Ackers, 2005, 2008; De Filippo y otros, 2007; Barjak y Robinson, 2008).

Recientemente, la Comisión Europea (CEC, 2008a) ha puesto mayor énfasis en la necesidad del fomento de la movilidad de corto plazo como instrumento para la integración del Espacio Europeo de Investigación (EEI) y para la cooperación de éste con terceros países de menor nivel de desarrollo. Se hace así referencia a las estancias de ida y vuelta o "lanzadera" (shuttle stays) (CEC, 2008a: 33) como forma de compatibilizar el desarrollo científico del EEI con el de los países con mayor escasez de recursos, o como instrumento para ayudar a la compatibilización de la vida profesional y familiar de los investigadores, teniendo en cuenta que las estancias breves van normalmente asociadas al mantenimiento del puesto de trabajo en el país de origen y no implican la vinculación al sistema impositivo o de seguridad social del país de acogida, ni altos costes de mudanza, etc. (Ackers, 2008).

La definición de una frontera temporal mínima o máxima para que la movilidad produzca los beneficios esperados en términos de creación y difusión de conocimiento y capital humano y social es necesariamente arbitraria. Sin embargo, este tipo de delimitaciones suelen ser necesarias para hacer operativo el análisis empírico. En el marco del presente trabajo, el “investigador móvil" se define como aquél que ha realizado alguna estancia de investigación en el extranjero, de entre una semana y dos años de duración, a lo largo de su carrera.

Los mecanismos mediante los cuales se produce el impacto de la movilidad en la actividad científica son complejos (OCDE, 2008) y difieren en función del tipo de personas, organizaciones y movilidades implicadas (CEC, 2001). Woolley y Turpin (2009) sostienen que las visitas temporales a otros centros de investigación son fundamentales en el proceso de formación de capital humano científico y técnico, entendido éste como la suma del capital cognitivo, social y de las habilidades científicas de los investigadores (Bozeman, 2008). Martín-Sempere y otros (1999) y Martín-Sempere y Rey-Rocha (2003) también subrayan la importancia de los desplazamientos temporales para el progreso científico. A su vez, De Filippo y otros (2009) ponen de manifiesto la conexión entre las estancias de investigación internacionales y la pertenencia a redes internacionales de colaboración y la productividad científica. En este trabajo, partimos de la relevancia que la literatura asigna a la movilidad temporal de los investigadores para analizar las dinámicas de este tipo de movilidad en la población de investigadores andaluces.

En España, como en el conjunto de la Unión Europea, coinciden la alarma por la salida de personal científico del país con la impresión generalizada de que la movilidad de los investigadores, tanto a escala nacional como internacional, es insuficiente. La alarma por la dificultad de atraer a algunos investigadores de 
vuelta al sistema nacional de investigación se hace patente a través de la prensa, de declaraciones de los responsables políticos y de los mismos investigadores. No nos consta, no obstante, que se haya elaborado ningún estudio sistemático acerca de los flujos de emigración de los investigadores españoles.

A pesar de tratarse de un fenómeno importante para entender la dinámica del sistema nacional de investigación, y a pesar de que se destina a los programas de movilidad un volumen creciente de fondos públicos, la movilidad de los investigadores sigue siendo un fenómeno poco explorado en nuestro país. Martín-Sempere y otros (1997) y Martín-Sempere y Rey Rocha (2003) han analizado los datos relativos a estancias en el extranjero financiadas por diversos programas del anterior Ministerio de Educación, Ciencia y Cultura, para los períodos 19841994 y 1999-2000 respectivamente. Estos estudios evaluaron el perfil de los investigadores participantes en los distintos programas analizados por género, edades, organismos de origen, países de destino y duración y financiación de las estancias.

García-Romero (2000) investiga el efecto de la estancia postdoctoral en la productividad científica, a partir de una encuesta realizada a 200 doctores españoles habiendo realizado estancias en el Reino Unido y llega a la conclusión de que tras la estancia postdoctoral se produce un sensible incremento de la productividad de los investigadores. De Filippo y otros (2007 y 2009) y De Filippo (2008) encuentran evidencia clara de relación entre la movilidad temporal de los investigadores de la Universidad Carlos III de Madrid y el número e impacto de sus publicaciones científicas en colaboración. Sin embargo, en nuestra investigación relativa al impacto de la movilidad sobre la producción científica para una muestra de 266 investigadores participantes en el programa Ramón y Cajal, no encontramos evidencia de relación entre movilidad y publicaciones sino más bien entre movilidad y participación en proyectos internacionales de investigación (Cañibano y otros, 2008). Sin dejar de ser relevantes, estos resultados no son del todo concluyentes ni generalizables a poblaciones más amplias de investigadores, aunque nos referiremos a ellos más adelante para compararlos con algunos de los indicadores que presentamos en este estudio.

La primera dificultad para el análisis de dinámicas de movilidad de los investigadores y del impacto de la misma, ha sido la escasez de datos, la cual es generalizada en Europa (CEC, 2008a y 2008b). Los estudios anteriormente referidos han empleado una variedad interesante de fuentes: información acerca de los solicitantes de ayudas a la movilidad facilitada por el Ministerio, datos procedentes de una encuesta, información contenida en la memorias de investigación de la Universidad e información contenida en los currículos de los investigadores remitidos por éstos mismos al Ministerio y entrevistas a los investigadores. Estas distintas metodologías tienen en común su laboriosidad, al requerir la extracción o codificación manual de los datos de la fuente original, lo cual explica el reducido tamaño de las muestras analizadas.

Los resultados que presentamos en este trabajo aplican nuevamente la metodología del análisis del curriculum vitae, pero introduciendo la novedad de que 
han sido directamente extraídos en formato electrónico del Sistema de Información Científica de Andalucía, sin necesidad de codificación manual, lo cual nos permite analizar los perfiles de movilidad temporal de toda la población de investigadores andaluces. Damos, por tanto, un salto metodológico importante que nos conduce a resultados que no están afectados por sesgos muestrales y que, por tanto, adquieren mayor relevancia y representatividad. Esto es posible gracias, por una parte al uso del curriculum vitae como fuente de información y, por otra, al desarrollo de sistemas de almacenaje y tratamiento de la información curricular que las nuevas tecnologías facilitan.

\section{Desarrollos metodológicos: la información curricular como herramienta para el análisis de la movilidad}

El currículum vitae es el único documento que resume todas las actividades profesionales de los investigadores y que registra la evolución actualizada de su trayectoria a lo largo del tiempo. Se trata, pues, de una valiosa fuente de información longitudinal, difícil de obtener en su totalidad a través de otras vías (Dietz y otros, 2000). A pesar del potencial que ofrecen, los currículos no empezaron a ser utilizados de forma sistemática como herramienta para la evaluación de la investigación y de la política científica hasta inicios de los años 2000. El equipo pionero en el empleo de esta metodología fue el de los miembros del proyecto "Research Value Mapping Programme", en la actualidad ubicado en la Universidad del Estado de Arizona ${ }^{2}$.

Los resultados alcanzados en el marco de este proyecto pusieron de manifiesto el potencial de los currículos como herramienta de trabajo para investigadores sociales e instituciones públicas interesados en el estudio o la evaluación de las dinámicas de investigación. El equipo empleó con éxito los currículos para estudiar las trayectorias profesionales de científicos e ingenieros, analizando, entre otras cuestiones, dinámicas de productividad y de colaboración con la industria (Gaughan y Bozeman, 2002; Corley y otros, 2003, Lee y Bozeman, 2005). Dado el carácter relativamente reciente del inicio en el empleo de esta metodología, ha sido posible realizar, en el marco de otro trabajo, una presentación del estado actual del arte en el uso de currículos para la evaluación de la investigación (Cañibano y Bozeman, 2009).

Los currículos también han sido empleados con éxito para analizar dinámicas de movilidad y del impacto de la misma en la productividad científica (Dietz y Bozeman, 2005; Cañibano y otros, 2008, De Filippo y otros, 2009, Sandström, 2009; Zubieta, 2009), en las trayectorias profesionales (Gaughan y Robin, 2004; Sabatier y otros, 2006), en las dinámicas de colaboración internacional (Fontes, 2007; Jonkers y Tijssen, 2008) y en la emergencia y desarrollo de redes de cooperación (Woolley y Turpin, 2009). Las movilidades estudiadas en estos trabajos

\footnotetext{
${ }^{2}$ http://www.cspo.org/rvm/
} 
son, salvo en el caso de los trabajos basados en el CV de investigadores españoles, los cambios de empleo y las migraciones. No nos consta la existencia de trabajos, en la literatura internacional, que hayan empleado currículos para el estudio y evaluación de la movilidad de corta duración de los investigadores, lo que constituye el objeto de este trabajo. Asimismo, toda la investigación realizada hasta la fecha a partir de esta metodología ha requerido la codificación manual de los currículos. Como ya hemos apuntado, la segunda novedad que introducimos en relación con la literatura disponible es la utilización de datos curriculares descargados directamente de un sistema electrónico. El siguiente apartado describe brevemente el sistema de información curricular en desarrollo en España, así como el Sistema de Información Científica de Andalucía, del que proceden los datos utilizados.

\section{Hacia un sistema nacional de información curricular}

El sistema español de investigación científica cuenta en la actualidad con dos características fundamentales, susceptibles de constituir la base para el desarrollo de potentes mecanismos de evaluación y seguimiento de la actividad de sus recursos humanos. Estas características son, por una parte, el alto grado de estandarización del currículum vitae de los investigadores y, por otra, la creciente digitalización de los datos curriculares. En pocos años, el sistema ha pasado de utilizar estándares de currículos impresos en papel, a basarse en documentos electrónicos de estos mismos estándares, los cuales finalmente han quedado prácticamente relegados por el desarrollo de plataformas informáticas para la introducción, almacenaje y manipulación de datos curriculares.

España ha sido uno de los países pioneros en el desarrollo de este tipo de plataformas y sistemas. La implantación paralela de varios tipos de sistemas de almacenamiento curricular en diversos organismos condujo, sin embargo, a la dispersión y falta de homogeneidad de los datos por una parte y, por otra, a una situación en que la repetida cumplimentación del currículo en diversas aplicaciones informáticas acabó suponiendo para los investigadores una carga mucho mayor que la de la actualización del habitual anterior documento de texto. La situación a la que nos referimos sería, por ejemplo, la de un investigador que debe cumplimentar su currículo en el sistema de gestión de datos curriculares de su universidad para obtener una bolsa de viaje y asistir a un congreso, a la vez debe introducir los datos de su currículo en la aplicación de la Agencia Nacional de Evaluación y Acreditación de la Calidad (ANECA) y en la correspondiente Agencia de Acreditación regional si desea ser acreditado. Por otra parte, debe también introducir sus datos curriculares en la correspondiente plataforma ministerial para concurrir a la obtención de fondos públicos de investigación, etc.

Es a esta situación a la que la Fundación Española para la Ciencia y la Tecnología (FECYT) quiso poner remedio con la puesta en marcha del proyecto CVN (currículum vitae normalizado), el cual culminó con éxito con el desarrollo de 
un estándar XML de comunicación y normalización para la interconexión de las bases de datos existentes (Báez y otros, 2008)³.

Entre las instituciones que en abril de 2009 ya habían implantado el CVN en sus sistemas se cuentan 13 universidades y la comunidad autónoma de Andalucía. La total implantación del nuevo formato normalizado y la consecuente total interconexión de los sistemas llevará todavía cierto tiempo. Una vez completado el proceso de adaptación, el potencial del nuevo sistema integrado será enorme, tanto por el volumen de información actualizada almacenado como por las posibilidades que brinda a investigadores, gestores y evaluadores para la utilización de la información curricular. Según la información facilitada por la FECYT, en abril de 2009 el número total de currículos disponibles en formato CVN ascendía a 30.440, lo que representa una cobertura del 25\% de la población nacional de investigadores. Según los últimos datos disponibles de la Encuesta de I+D del Instituto Nacional de Estadística, el total de investigadores en equivalencia a jornada completa asciende a 122.624 .

El desarrollo de este sistema nacional integrado de información curricular coloca a España en una situación privilegiada en comparación con la mayoría de los países. Sin duda se trata del país pionero en la Unión Europea en el desarrollo de este tipo de sistemas. Únicamente Portugal y Noruega están desarrollando sistemas de información curricular similares (Fontes, 2008; Ekeland, 2008). En América Latina, este tipo de sistemas están también en desarrollo en diversos países, entre los que se cuentan Argentina, Brasil o Colombia (D’Onofrio, 2009).

Como hemos apuntado anteriormente, los datos empleados en este trabajo proceden de uno de los sistemas de información que integran el conjunto del sistema nacional en construcción. Se trata del Sistema de Información Científica de Andalucía, el cual fue pionero en España (Solís Cabrera, 2008) y del que a continuación detallamos algunas características. La elección de este sistema para la realización del análisis se justifica por su ya larga trayectoria (operativo desde 2001) y su alto nivel de fiabilidad y cobertura.

\subsection{El Sistema de Información Científica de Andalucia: SICA}

SICA es un sistema global de información científica certificada en tiempo real. Se trata de un sistema de ámbito regional que agrupa información sobre la producción y la actividad científica. A medida que la información se genera, es introducida y validada, facilitando el análisis de las políticas científicas.

El Sistema se creó en 2001, con el objeto de adquirir, almacenar, organizar y recuperar la información relacionada con la actividad y la producción científicotécnica de los investigadores adscritos a centros de investigación, instituciones o universidades andaluzas. En este sentido, el sistema recoge información de todo el personal investigador del sistema de investigación andaluz. Con la recopilación

\footnotetext{
3 Para una descripción del proyecto CVN véase http://cv.normalizado.org/index.jsp.
}

Rev. Esp. Doc. Cient., 33, 3, julio-septiembre, 428-457, 2010. ISSN: 0210-0614. doi:10.3989/redc.2010.3.736 
y tratamiento de esta información se pretendía, entre otros objetivos, ayudar a la administración y en particular a los responsables de política científica en la toma de decisiones, y constituir una base de conocimiento autorizada en materia de evaluación y calidad de los resultados de la actividad científica. A día de hoy, SICA se ha convertido en una herramienta básica en la planificación, gestión y evaluación en políticas de ciencia y tecnología e innovación en la región (figura 1).

FIGURA 1

Estructura del sistema: agentes y resultados de investigación

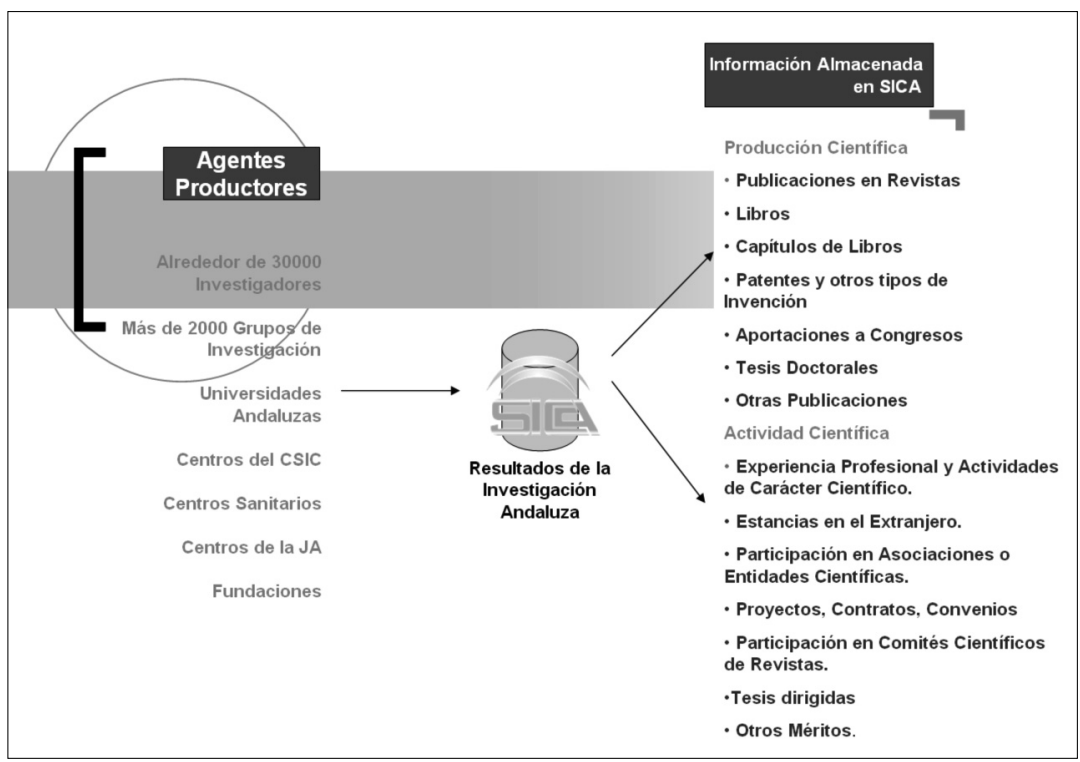

Fuente: SICA, 2009.

Cabe resaltar que el módulo de Gestión Documental, es una potente herramienta, que permite comprobar la veracidad y coherencia de la información mediante procedimientos como validación de la producción científica, detección de duplicados, cargas automáticas masivas procedentes de fuentes comerciales, control de autoridades, etc. Al mismo tiempo, complementa la labor de actualización del currículum de los investigadores y reduce el trabajo manual de grabar datos.

Hacemos hincapié en lo que consideramos han sido algunos de los factores de éxito de SICA:

- Compatibilidad de las salidas de información resultante con otros sistemas de información a nivel nacional. Cabe destacar que SICA ha sido el primer sistema en implementar un formato de salida del curriculum de los investigadores bajo la norma CVN (Currículum Vitae Normalizado). 
- Información accesible, actualizada, fiable y útil para los usuarios del sistema.

- Motivación de los investigadores y grupos de investigación para mantener actualizados sus perfiles de actividad científica.

- Utilidad del sistema como herramienta de consulta para trazar estrategias y políticas en materia de $\mathrm{I}+\mathrm{D}+\mathrm{i}$.

- La información que contiene brinda la posibilidad de servir como base material de estudio a investigaciones en el campo de la medición de la ciencia.

- Buena parte de la información se incorpora automáticamente ya validada, siendo descargada de bases de datos internacionales como SCOPUS, ISI, MEDLINE, SCIENCE DIRECT y las del CINDOC. El resto de la información que no aparece en estas bases las introduce el propio investigador. A continuación, el personal técnico del sistema valida la información introducida.

Todas las características anteriores hacen que, tal y como hemos apuntado, la fiabilidad del sistema sea muy alta. El análisis de algunos de los datos que contiene presenta, no obstante, obstáculos de carácter metodológico, susceptibles de ser resueltos en el futuro. No nos detendremos en la descripción de los mismos, dado que no son relevantes para el análisis aquí presentado. El estudio de las variables analizadas en este trabajo no supuso más que un sencillo trabajo de limpieza que se describe en el siguiente apartado.

\section{Movilidad temporal de los investigadores andaluces}

\subsection{Población}

El sistema de investigación andaluz emplea aproximadamente al 20\% de la población nacional de investigadores, entendiendo en este caso por «investigadores" a las personas que cumplen los requisitos descritos en la definición provista por el Manual de Frascati (OCDE, 2002) y aplicada por la encuesta nacional de actividades de I+D. En el contexto del estudio que nos ocupa, son considerados "investigadores" todas aquellas personas registradas como tales en el SICA, lo que incluye al personal docente investigador de las universidades públicas y privadas, los investigadores de centros de investigación de diversa índole y los investigadores del sector empresarial registrados en el sistema. El SICA tiene una cobertura de prácticamente la totalidad de la población investigadora andaluza, contando en mayo de 2009 con el curriculum de 30.737 investigadores. La inmensa mayoría de los investigadores andaluces trabaja en universidades $(76 \%$ del total). Asimismo, al considerar la distribución por áreas de conocimiento, resulta llamativo el peso de las humanidades (27\%) en comparación con el resto de áreas.

Del total de la población de investigadores andaluces, la sub-población objeto de estudio es aquella cuyo currículo registra alguna Estancia en el Extranjero. El currículum incluye un apartado para el registro de estancias temporales en 
centros extranjeros de investigación, pero no especifica una definición de "estancia" ni de las actividades vinculadas a las mismas (participación en congresos, cursos, estancias por proyectos de investigación, de formación, etc.). Esto es así en la mayoría de formatos estandarizados de currículos de investigadores en España, facilitados por diversas instituciones, lo que deja en manos del investigador la decisión de qué considera como "estancia". El currículo del SICA registra el organismo de destino de la estancia (y por tanto, el país), la fecha de inicio y de finalización de la misma e incluye una variable de "tipo de estancia" que presenta problemas metodológicos y que por tanto no hemos podido emplear en este trabajo. La variable "tipo de estancia" del sistema, ofrece un menú de opciones que no son mutuamente excluyentes y que son poco informativas (becario, contratado, doctorado, invitado, otras y postdoctoral). A raíz de la elaboración de este estudio, se ha aconsejado a los administradores del SICA que, en el nuevo diseño en curso del sistema, se resuelva este problema que invalida la utilidad de esta información.

Es el investigador quien decide cómo cumplimentar este apartado y qué tipo de estancias o movimientos incluir en él. En total, en la población cuyos datos se descargaron contamos 6.955 investigadores que realizan 20.990 estancias, a lo largo del período 1961-2009. Los datos analizados fueron descargados del sistema en marzo de 2009. Al tratarse de un sistema dinámico, la población de investigadores y la totalidad de estancias en el sistema esta lógicamente en constante cambio.

De este total se eliminan, para realizar el estudio, los registros con campos vacíos (por ejemplo estancias sin fecha de finalización), o con campos con valores incorrectos (por ejemplo, fechas de nacimiento erróneas). Al tratarse de muy pocos casos, esto no distorsiona los resultados del trabajo. Eliminamos también las estancias con una duración superior a dos años, al ser éstas poco numerosas y no encajar con lo que normalmente se entiende por "estancia de investigación". Se trataría más bien de una movilidad que en muchos casos deriva de que los investigadores registran como estancias la realización del doctorado en el extranjero o la duración íntegra de algunos contratos de trabajo.

Del mismo modo, no consideramos las estancias inferiores a una semana de duración, con objeto de evitar la confusión de las mismas con la participación en congresos o con la colaboración docente puntual, normalmente consistente en la impartición de algunas clases a lo largo de 1 o dos días. Tal y como hemos mencionado anteriormente, estas decisiones en la delimitación temporal a la hora de seleccionar la información son arbitrarias y se basan en nuestra experiencia anterior en la codificación manual de currículos. Puede darse el caso de que una visita muy breve dé frutos importantes en términos de establecimiento de lazos de colaboración, pero al no considerar que ésta sea la norma, preferimos excluirlas. Las estancias de duración inferior a una semana representan el 10,6\% del total. Por último, se eliminan las estancias registradas en este apartado del currículo pero realizadas en España, al no tratarse de movilidad internacional. El número total resultante de investigadores analizados es de 6.345 y el número de estancias de 17.643, por lo que la sub-población final analizada se compone 
aproximadamente del $91 \%$ de la población total que declara estancias en su currículo.

La población analizada se caracteriza por un peso mayor de los investigadores universitarios (88\%), por un mayor peso de los hombres (60\%) en comparación con las mujeres, por el predominio del grupo de edad comprendido entre 30 y 40 años (43\%) y por un porcentaje de doctores del 70\%, en comparación con los no-doctores. Con respecto a las áreas de conocimiento, igual que para el conjunto de la población, son las humanidades el área más representada (30\%), seguida por las ciencias sociales, económicas y jurídicas (18\%) y por la física, química y matemáticas (11\%). Estas proporciones, así como el detalle de las 9 áreas de conocimiento en las que el SICA clasifica las distintas disciplinas científicas, quedan reflejadas en la tabla I.

\section{TABLA I}

Distribución de la población analizada por área de conocimiento

\begin{tabular}{c|c|c|c|c|c}
\hline $\begin{array}{c}\text { Código } \\
\text { disciplina }\end{array}$ & $\begin{array}{c}\text { Frecuencia } \\
\text { absoluta }\end{array}$ & $\begin{array}{c}\text { Frecuencia } \\
\text { relativa } \\
\mathbf{( \% )}\end{array}$ & $\begin{array}{c}\text { Frecuencia } \\
\text { absoluta } \\
\text { acumulada }\end{array}$ & $\begin{array}{c}\text { Frecuencia } \\
\text { relativa } \\
\text { acumulada } \\
\mathbf{( \% )}\end{array}$ & Descripción disciplina \\
\hline HUM & 1.896 & 29,88 & 1.896 & 29,88 & HUM = Humanidades \\
\hline SEJ & 1.110 & 17,49 & 3.006 & 47,38 & $\begin{array}{c}\text { SEJ = Ciencias Económicas, Socia- } \\
\text { les y Jurídicas }\end{array}$ \\
\hline FQM & 719 & 11,33 & 3.725 & 58,71 & $\begin{array}{c}\text { FQM = Física, Química y Matemá- } \\
\text { ticas }\end{array}$ \\
\hline TEP & 339 & 5,34 & 4.064 & 64,05 & $\begin{array}{c}\text { TEP = Tecnologías de la produc- } \\
\text { ción }\end{array}$ \\
\hline RIO & 440 & 6,93 & 4.504 & 70,99 & BIO = Biología y Biotecnología \\
\hline CTS & 443 & 6,98 & 5.537 & 87,27 & $\begin{array}{c}\text { CTS = Ciencia y Tecnología de la } \\
\text { Salud }\end{array}$ \\
\hline TIC & 412 & 6,49 & 5.949 & 93,76 & $\begin{array}{c}\text { TIC = Tecnologías de la Informa- } \\
\text { ción y de las Comunicaciones }\end{array}$ \\
\hline AGR & 396 & $6 \%$ & 6.345 & 100,00 & AGR = Agroalimentación \\
\hline
\end{tabular}

\subsection{Estancias temporales: visión de conjunto}

Uno de los primeros resultados que salta a la vista es el hecho de que la población con estancias representa el 22\% del total, lo que significa que las tres cuartas partes de la población analizada no ha realizado (o no ha declarado) ninguna estancia en el extranjero. Nuestros resultados no son directamente com- 
parables con los escasos datos existentes en España procedentes de estudios anteriores, pero sí se registran coincidencias que merece señalar. El trabajo de De Filippo (2008) registra un $22 \%$ de investigadores con estancias en otros centros (incluyendo centros nacionales e internacionales) en la población analizada. De Filippo y otros, (2007) encuentran un promedio de participación en programas de movilidad del 8\% de la población analizada y un promedio de participación en los programas de movilidad del Ministerio de Educación y Ciencia del $1,52 \%$, porcentajes que oscilan entre el $0,6 \%$ y el $2 \%$ en el trabajo de MartínSempere y Rey Rocha (2003). En nuestro caso, al no disponer de la información relativa a los programas a los que corresponden las estancias, no es posible realizar una estimación comparativa. Como puede apreciarse en la figura 2, el 64\% del total de 17.643 estancias estudiadas tiene una duración inferior o igual a tres meses, por lo que destacan sobremanera las estancias breves de investigación sobre otras de mayor duración. Aproximadamente el 29\% tiene una duración de entre 3 y 12 meses y otro $7 \%$ de entre 12 y 24 meses. Estos resultados también son coincidentes con los de De Filippo (2008) y De Fillipo y otros (2009), quienes destacan que la mayor parte de las estancias analizadas tiene una duración de 1 a 3 meses. Estos resultados no son comparables con los resultados de Martín Sempere y Rey Rocha (2003), quienes analizan las estancias enmarcadas por el programa del anterior Ministerio de Educación y Ciencia de Investigadores Españoles en el Extranjero. El programa financia estancias de 1 a 24 meses de duración siendo el promedio de duración de las mismas de unos 9 meses, oscilando en función de las áreas de conocimiento. Parece razonable pensar que los investigadores que planean una estancia de solo algunas semanas prefieran no hacer el esfuerzo de presentar una solicitud a este programa, más orientado a la financiación de estancias más largas. Esto justificaría también que tanto la proporción de solicitudes como de becas obtenidas sea mayor en el área de ciencia y tecnología que en ciencias sociales y humanas $(60 \%$ y $40 \%$ respectivamente en los años estudiados). Como tendremos ocasión de apuntar más adelante, nuestro estudio revela una tendencia a realizar estancias de menor duración en las ciencias sociales y humanas que en otras disciplinas.

Cabe destacar que, aún sin ser directamente comparable a nuestro estudio, una encuesta reciente encargada por la Comisión Europea con objeto de analizar los obstáculos a la movilidad de los investigadores europeos, alcanza un resultado de 24\% de investigadores móviles sobre el total de 3.365 investigadores encuestados. En el caso de este estudio europeo (CEC, 2008b), el investigador móvil se define como aquel residente en un país distinto a su país de origen en el momento de contestar la encuesta. En el marco de nuestro trabajo, el "investigador móvil" es aquél que ha realizado alguna estancia de investigación en el extranjero a lo largo de su carrera. Se trata de conceptos diferentes pero en ambos casos se obtiene un resultado de aproximadamente una cuarta parte de la población (figura 2).

La figura 3 muestra la distribución del número de estancias por investigador, poniendo de relieve que la mayoría (el 40\%) declara únicamente una estancia a 
FIGURA 2

Distribución de estancias por duración

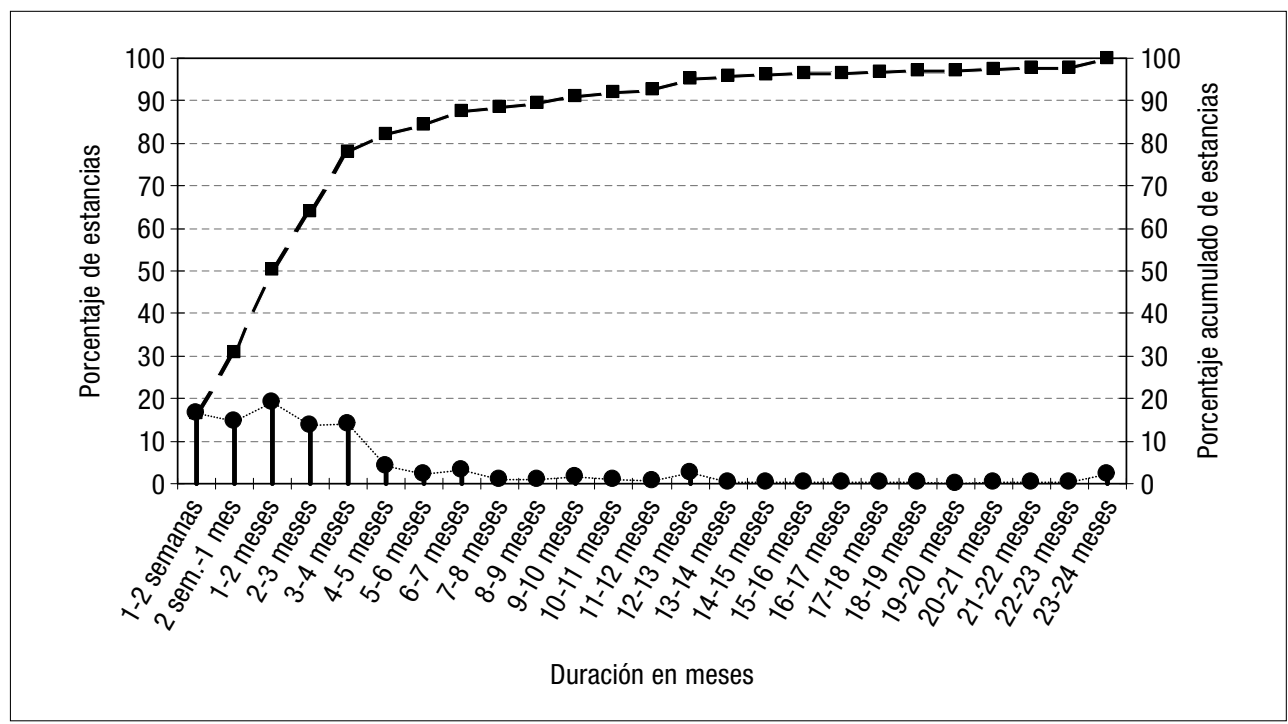

FIGURA 3

Distribución de estancias por investigador

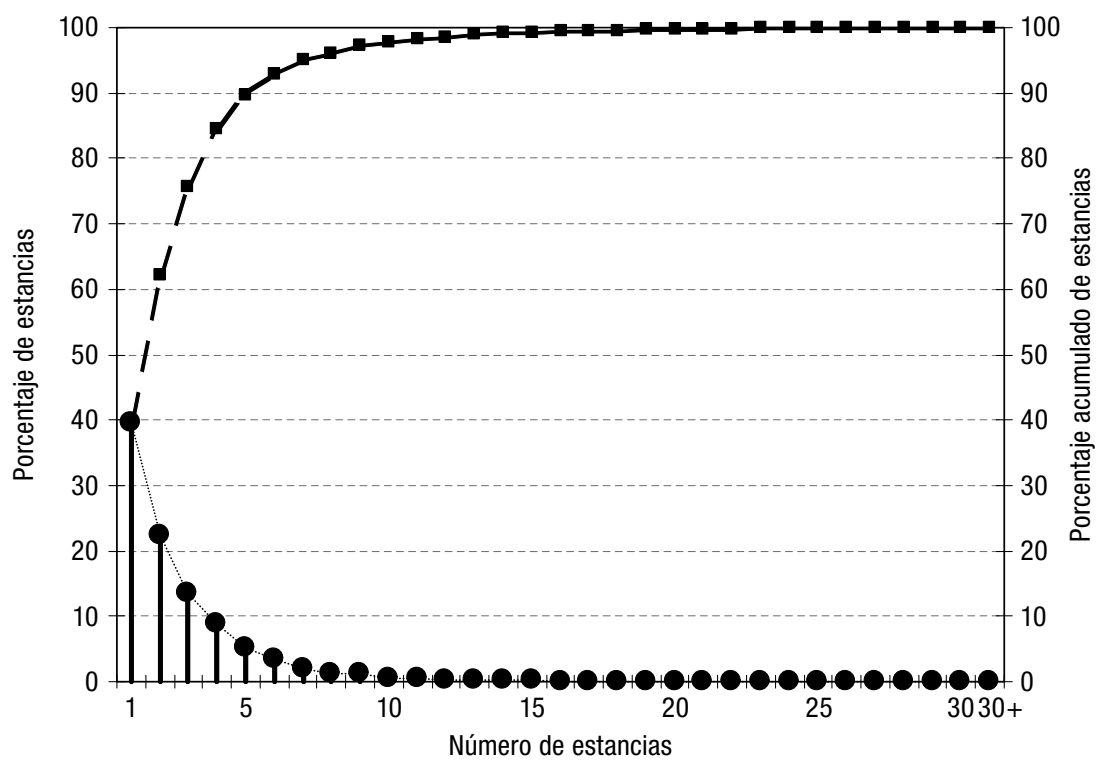


lo largo de su carrera. El 90\% ha realizado entre 1 y 5 estancias. En el trabajo de De Filippo (2008) el porcentaje de investigadores con 1 estancia de investigación asciende a $61 \%$ de la población estudiada.

En relación con el destino geográfico de las estancias, Europa Occidental agrupa el 61\% de las estancias, seguida de América Latina con el 17\% y de Norteamérica (15\%). Puede apreciarse en la figura 4 como los países más visitados, es decir, Reino Unido, Alemania, Francia e Italia, junto con Estados Unidos, agrupan el 60\% del total de estancias. De Filippo (2008) obtiene un 66\% de estancias que tienen como destino estos 5 países. Martín Sempere y Rey Rocha (2003) obtienen un porcentaje de $81 \%$, lo que podría explicarse por el menor peso de los investigadores de ciencias sociales y humanas en la población que analizan.

Cabe destacar, no obstante, que son México, Portugal y Argentina, los tres países que siguen a estos destinos en importancia. La evolución de los destinos a lo largo del tiempo resulta especialmente interesante. Lo comentaremos más adelante en la sección relativa al análisis dinámico de las estancias.

Con respecto al análisis por género para el conjunto de la población con estancias, aparecen diferencias significativas ( $p$-valor $=0,0084)$ a favor de una mayor movilidad de los hombres, si se compara la proporción de mujeres in-

\section{FIGURA 4}

Estancias por países más visitados

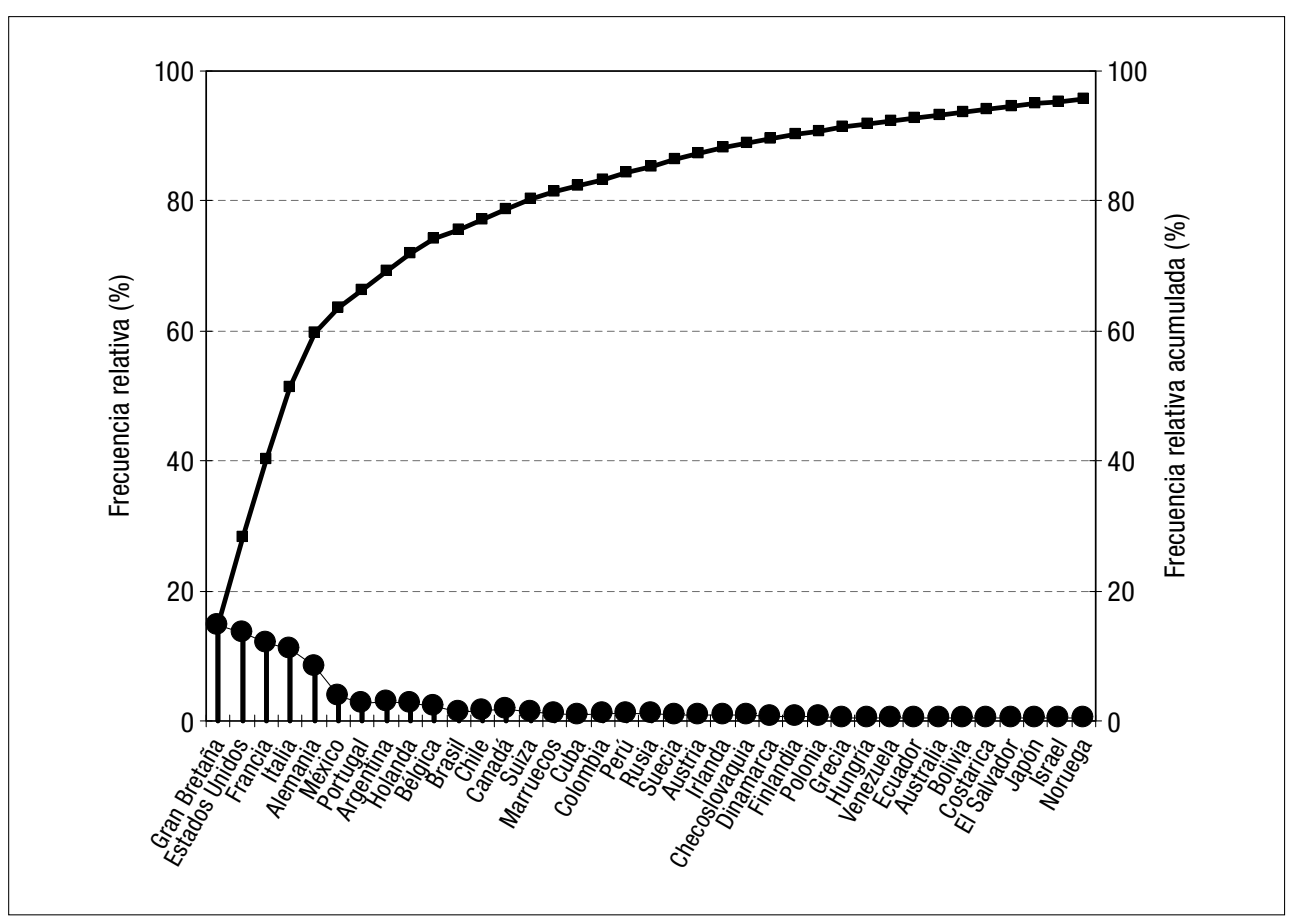

Rev. Esp. Doc. Cient., 33, 3, julio-septiembre, 428-457, 2010. ISSN: 0210-0614. doi:10.3989/redc.2010.3.736 
vestigadoras $(40,41 \%)$ con la proporción de estancias realizadas por mujeres $(38,52 \%)$. Estas diferencias dejan de ser significativas para las cohortes más jóvenes de población, de modo que no puede apreciarse realmente una diferencia por género en las dinámicas de movilidad temporal de los investigadores jóvenes (25 a 40 años). De Filippo y otros, (2009) encuentran una mayor proporción de mujeres con estancias que de hombres, lo que achacan a la alta proporción de mujeres jóvenes en la población. Las mujeres con movilidad en esta población son más jóvenes que las que no declaran estancias. Por su parte, Martín- Sempere y Rey Rocha (2003) encuentran diferencias importantes de género tanto entre los solicitantes de las becas de movilidad como entre aquellos que las obtuvieron, siendo la proporción de hombres sensiblemente mayor que la de mujeres. Las convocatorias que estos autores analizan son las de 1999 y 2000. Aunque los resultados de estos estudios no son directamente comparables, la combinación de evidencias parece apuntar a que la incorporación de mujeres al sistema de investigación a lo largo de la última década ha ido eliminando las diferencias de género en las dinámicas de movilidad entre las cohortes más jóvenes.

\subsection{Estancias temporales: evolución}

No nos consta ningún otro estudio que analice la evolución de estancias de investigación a lo largo del tiempo en España, por lo que en este apartado no podemos contrastar nuestros resultados con otros, tal y se ha hecho para el análisis estático.

El número absoluto de estancias en el extranjero ha crecido sustancialmente a lo largo del tiempo, lo cual es fruto, en gran medida, del aumento de la población de investigadores. Estimamos la evolución en el número de estancias por investigador y año, dividiendo el número de estancias para cada año por la población mayor de 25 años en ese año, edad a partir de la cual entendemos que es posible que un investigador empiece a ser móvil, normalmente en el marco de sus estudios de doctorado. La figura 5 muestra cómo las estancias por investigador han ido creciendo de forma continua desde mediados de la década de 1980, resultado que necesariamente hemos de vincular a la puesta en marcha de las primeras acciones de fomento de la movilidad a mediados de los ochenta, a la entrada en vigor de la Ley de la Ciencia en 1986 y, más recientemente, a la proliferación de instrumentos de apoyo a la movilidad temporal y a la creciente internacionalización de la práctica científica. Achacamos la disminución registrada en los últimos años de la serie a la posibilidad de que muchos investigadores no hayan actualizado aún en el sistema la información relativa a estancias más recientes.

Al margen de la tendencia registrada, cabe destacar que el máximo alcanzado por la serie es de aproximadamente un cuarto de estancia por investigador, lo que implicaría una media de una estancia cada 4 años.

Rev. Esp. Doc. Cient., 33, 3, julio-septiembre, 428-457, 2010. ISSN: 0210-0614. doi:10.3989/redc.2010.3.736 
FIGURA 5

Estancias por investigador y año

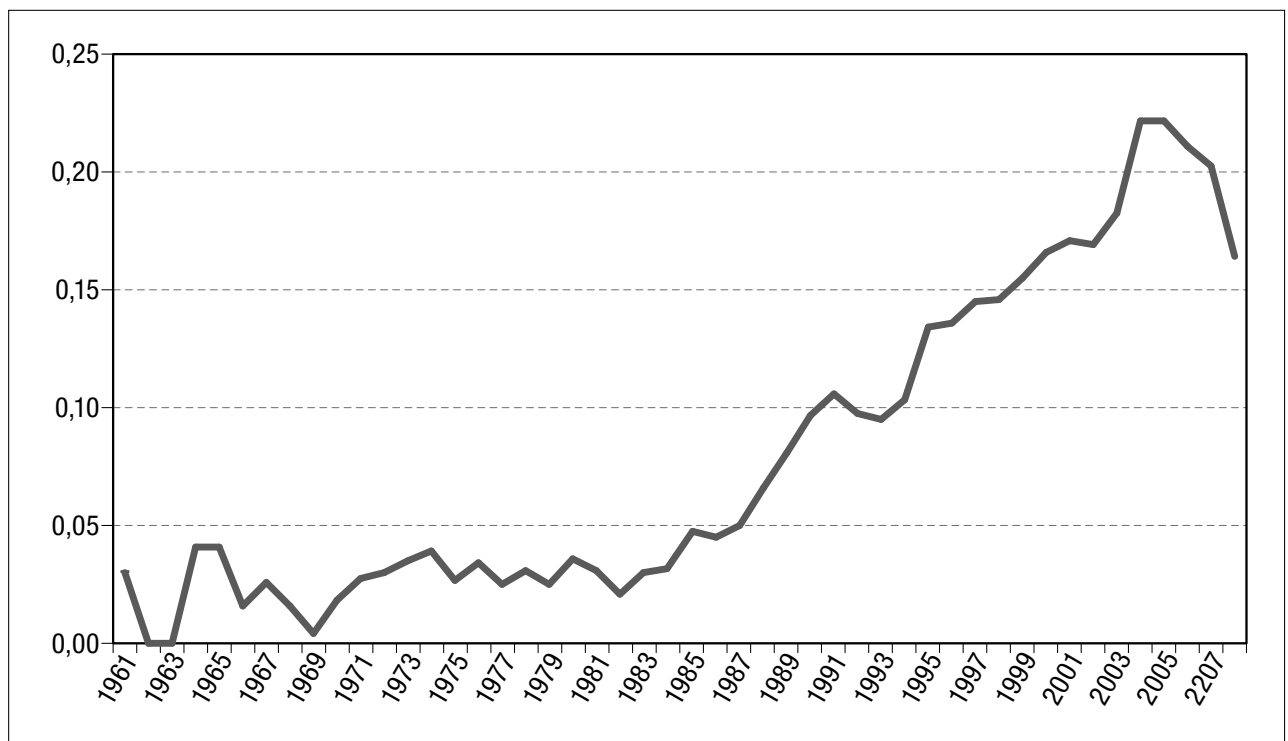

Con objeto de profundizar en el análisis de la evolución de las estancias, nos centramos en la serie temporal 1970-2010 y la dividimos en cuatro períodos, clasificamos asimismo los países de destino de las estancias en zonas geográficas. El último período abarca la década 2000-2010 teniendo en cuenta que, aunque sólo se han considerado estancias registradas en el sistema hasta 2009, algunas de ellas tienen como año de finalización 2010. Se registran cambios significativos en las zonas de destino, con la emergencia de África y América Latina a partir de 2000, a expensas de una disminución relativa de Estados Unidos y Europa Occidental. La realización de un análisis bifactorial de las variables "década" y "zona", una vez ajustadas las comparaciones según los tamaños muestrales, confirma estas tendencias. Los signos de la tabla II ponen de manifiesto las variacio-

TABLA II

Destinos por década

\begin{tabular}{c|c|c|c|c|c}
\hline Década & Latinoamérica & $\begin{array}{c}\text { Europa } \\
\text { Occidental }\end{array}$ & Norteamérica & África & $\begin{array}{c}\text { Europa } \\
\text { del Este }\end{array}$ \\
\hline $2000-2010$ & + & - & - & + & + \\
\hline $1990-1999$ & - & + & + & - & \\
\hline $1980-1989$ & - & & + & & \\
\hline $1970-1979$ & - & & & & \\
\hline
\end{tabular}


nes significativas en la preferencia relativa de cada zona con respecto a las demás, para cada década. Destacan, por tanto, los cambios registrados en la última década con el crecimiento significativo de nuevos destinos geográficos y la disminución en el peso de los destinos tradicionales.

La figura 6 muestra el resultado de analizar conjuntamente las variables país y década en las que se realiza la estancia. Figuran sólo los países con un comportamiento significativamente distinto en alguna década con respecto a su comportamiento en general, indicando en el eje de ordenadas el valor tipificado de la diferencia entre el porcentaje para una década y el porcentaje general. Cabe destacar la emergencia de Finlandia, Portugal y México en la última década en contraste con la significativa disminución registrada en algunos de los destinos tradicionales, como Reino Unido, Francia o Estados Unidos. Este análisis se llevó a cabo también incluyendo las estancias de duración inferior a una semana. La diferencia encontrada con respecto a los resultados presentados es la presencia de Marruecos como otro destino emergente en la última década. La cercanía de este país con Andalucía podría explicar la emergencia de dinámicas de colaboración basadas en visitas muy breves.

FIGURA 6

Países que registran cambios significativos por década

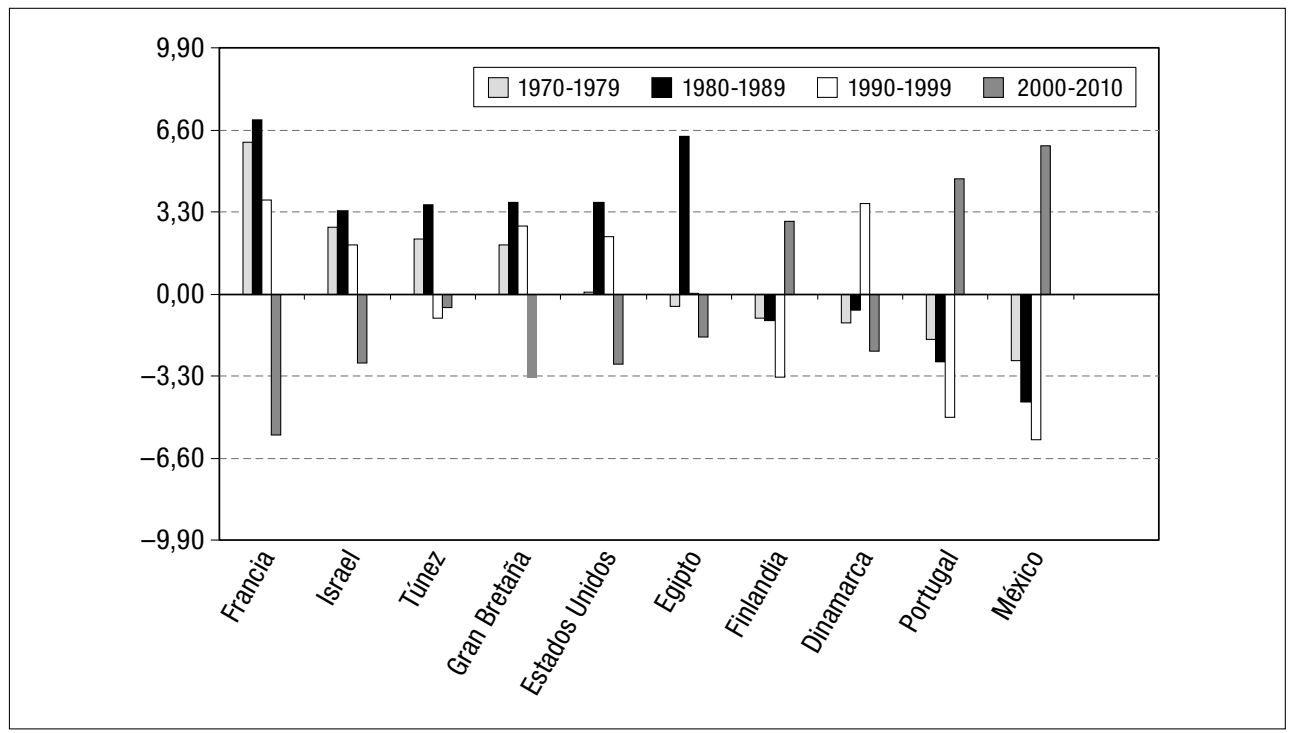

\subsection{Perfiles de movilidad temporal por área de conocimiento}

La clasificación por áreas de conocimiento empleada se corresponde con las 9 grandes áreas en que SICA clasifica a investigadores y grupos de investigación, 
al igual que en la tabla I. Los perfiles de actividad investigadora difieren notablemente entre áreas de conocimiento. Aparte de las ya conocidas diferencias en las dinámicas de producción científica, la literatura también ha puesto recientemente de manifiesto diferencias en las dinámicas de movilidad por área de conocimiento. Zubieta (2009) detecta marcadas diferencias en las dinámicas de movilidad postdoctoral entre las áreas de ciencias puras y las de ingeniería. Anteriormente, nosotros también encontramos diferencias significativas en la movilidad de investigadores de biología molecular, física y ciencias del espacio y filosofía y filología (Cañibano y otros, 2008). No obstante, debido a los tamaños muestrales y a las limitaciones metodológicas apuntadas con anterioridad en este trabajo, los resultados hallados hasta la fecha, aunque indicativos de ciertas dinámicas, no pueden considerarse representativos de ninguna población. Nos disponemos, por tanto, a estudiar las posibles diferencias existentes entre las pautas de movilidad temporal de las distintas áreas de conocimiento en la población de investigadores andaluces objeto de estudio.

La síntesis de resultados que presentamos a continuación está contenida en la tabla III. Las comparaciones se han realizado según diversos contrastes estadísticos. Para comparar las distribuciones de edad, de la duración en meses de las estancias, del número de estancias por investigador, de las zonas visitadas y de los períodos temporales, se ha realizado un contraste de bondad de ajuste a la distribución de la población. Si el contraste detecta falta de ajuste, se realiza un contraste de proporciones para cada categoría para identificar los valores significativamente mayores (los valores con fondo negro en la tabla III) o menores (los valores con fondo gris) que la proporción para el conjunto de la población. Para comparar el porcentaje de hombres o de doctores con respecto a la media se utiliza también el contraste de proporciones y para la media de estancias por investigador un contraste de medias. Para el estudio de género se realiza, además, un contraste de diferencia de proporciones para determinar si el grupo de hombres es más móvil que el de las mujeres. Se proporciona el valor de la diferencia tipificado, indicando los valores positivos, mayor movilidad en los hombres y los negativos lo contrario.

\section{Características socio-demográficas}

Puede apreciarse en la tabla III cómo el porcentaje de hombres es sistemáticamente mayor que el de mujeres para todas las áreas de conocimiento (cabe recordar que estamos analizando la población móvil o con estancias internacionales y no el total de la población de investigadores andaluces). Destacan como significativamente mayores al resto de las áreas los porcentajes de hombres en Física, Química y Matemáticas (FQM), Tecnologías de la Producción (TEP) y Tecnologías de la Información y la Comunicación (TIC). En ciencias de la Agricultura (AGR) y Humanidades (HUM) en cambio, el porcentaje de hombres es significativamente inferior al del resto de las áreas. 


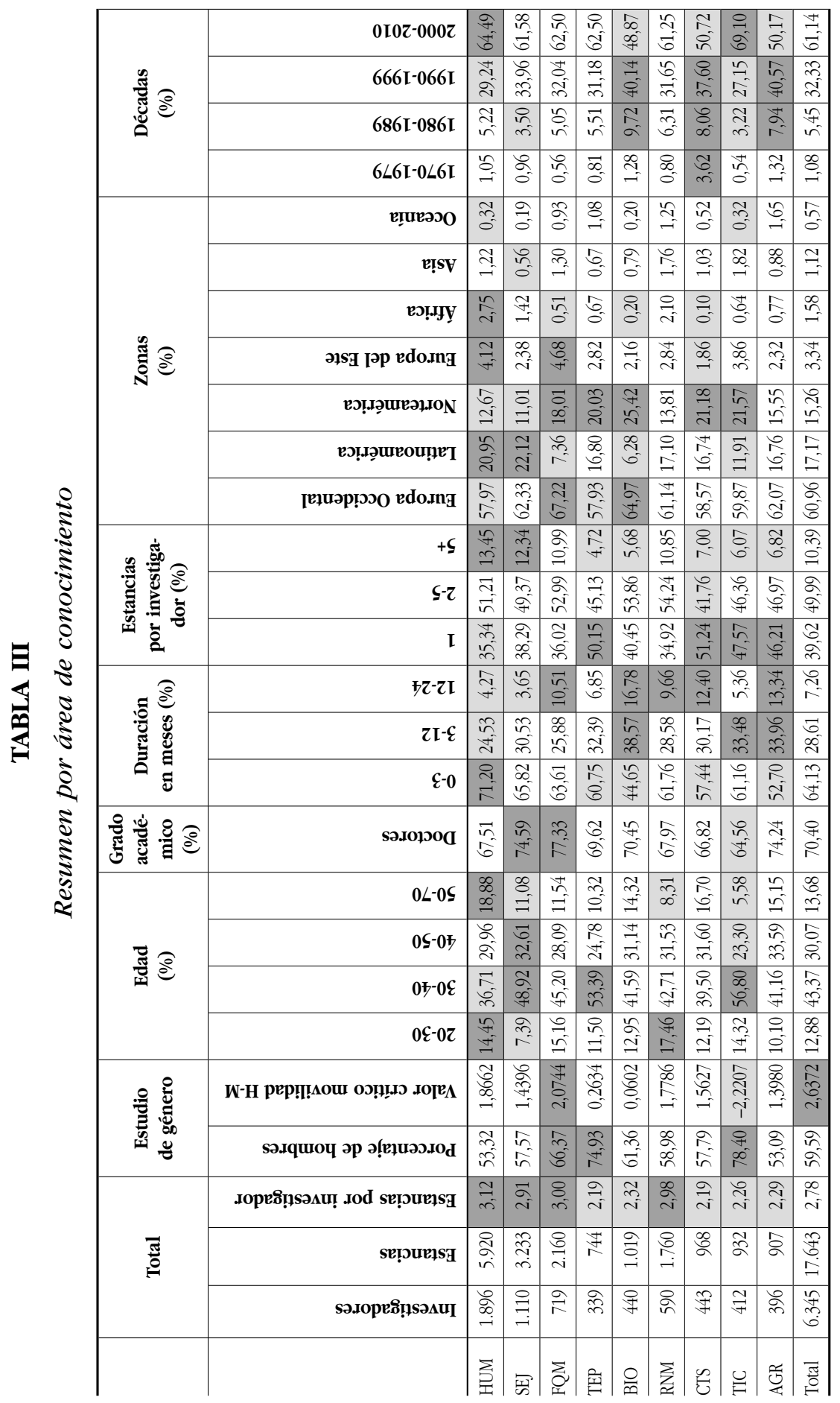


El análisis del conjunto de la población pone de manifiesto que los hombres son más móviles que las mujeres en todas las áreas de conocimiento excepto en TIC. Sin embargo, al tener en cuenta los grupos de edad, estos resultados cambian, tal y como apuntamos anteriormente en relación con el análisis de género. El porcentaje de estancias realizadas por hombres disminuye a medida que disminuye la edad, mientras que aumenta la movilidad de las mujeres. Para el grupo de edad comprendido entre 20 y 30 años, el porcentaje de estancias realizadas por mujeres fue superior en AGR, Biología (BIO), HUM y Recursos Naturales y Medioambiente (RNM). En el grupo de edad de 30 a 40 años, realizaron más estancias las mujeres que los hombres en AGR, HUM y Ciencias Económicas Jurídicas y Sociales (SEJ). Estas diferencias entre géneros no son aún estadísticamente significativas pero muestran un cambio de tendencia hacia la igualdad.

Con respecto a la distribución por edades, comprobamos cómo el grupo de edad comprendido entre 30 y 40 años es el más numeroso en todas las áreas de conocimiento, seguido por el grupo comprendido entre 40 y 50 años. Destaca el área de RNM, con una población significativamente más joven que en las demás áreas (un peso significativamente mayor del grupo más joven y significativamente menor del grupo mayor de 50). HUM llama la atención por el mayor peso de la población en los extremos de la pirámide de población, es decir, del grupo más joven por una parte y del grupo mayor de 50 por otra. Contrasta con esta distribución el área de SEJ, con una concentración de población significativamente mayor en los grupos medios (de 30 a 50) y menor en los extremos.

Finalmente, se aprecia cómo el porcentaje de doctores es superior al de no doctores en todas las áreas (superior en todos los casos al 60\%), con un peso significativamente mayor en SEJ (75\%) y FQM (77\%) y menor en TIC (65\%). Cabe resaltar que, en esta última área, el 37\% de la población móvil está por tanto compuesta por no doctores y que, en términos medios, éstos representan un 30\% del total de la población con estancias, lo cual refleja la importancia de las estancias temporales pre-doctorales.

\section{Perfiles de movilidad por área}

- HUM: Humanidades (1.896 investigadores)

A lo largo del período considerado, las estancias por investigador han crecido en las Humanidades por encima de la media, lo que resulta en una mayor concentración del total de estancias realizadas en la última década. La proporción de estancias realizadas en el período 2000-2010 asciende a 64,49\%, frente a un 61,14\% para el conjunto de la población. Sólo el área de TIC supera este porcentaje en la última década, con un 69,10\%. Humanidades se caracteriza, en términos generales, por una movilidad más frecuente y menos duradera que el resto de las áreas. Destaca el mayor número de estancias, las cuales representan un 33,55\% del total, y de estancias por investigador $(13,45 \%$ con más de 5 estancias frente una media de 10,39\%). Destaca también el peso significativamen- 
te mayor de las estancias de 1 semana a 3 meses de duración (71,20\% frente a un promedio de 64,13\%). Es asimismo, junto con el área de Ciencias Económicas Sociales y Jurídicas, la principal responsable de la emergencia de América Latina como nueva región de destino (el peso de esta área en las estancias que tuvieron como destino México, Argentina, Chile y Cuba, entre otros países Latinoamericanos, es especialmente alto: 20,95\% para el conjunto de la región frente a un promedio de $17,7 \%$ ). Asimismo, los investigadores de HUM van significativamente más a África que los de las demás disciplinas (destacando Egipto y Marruecos). Aún siendo Europa Occidental el principal destino (57,97\% de las estancias), los investigadores de Humanidades se desplazan significativamente menos a esta región y a Norteamérica que los de las demás áreas, y más a las regiones emergentes anteriormente mencionadas, especialmente a lo largo de la última década.

- SEJ: Ciencias Económicas Sociales y Jurídicas (1.110 investigadores)

El perfil de esta área es similar al de las Humanidades. Se caracteriza por un número de estancias total y por investigador superiores a la media, siendo éstas principalmente de duración corta (de 1 semana a 3 meses). Como en el caso de las Humanidades, las estancias superiores a 12 meses son significativamente inferiores al resto de disciplinas. En lo que se refiere a los destinos geográficos, se trata del área con mayor proporción de estancias en América Latina y menor en Norteamérica.

\section{- FQM: Física-Química-Matemáticas (719 investigadores)}

La tercera disciplina en importancia, según el número total de investigadores también presenta un número de estancias por investigador superior a la media (3 frente a una media de 2,78) pero presenta algunas pautas distintas a las de los grupos anteriores. La frecuencia de las estancias de corta duración, aún siendo alta $(63,61 \%)$, es menor que en estos grupos, mientras que es significativamente superior a la media el peso de las estancias de 12 a 24 meses de duración $(10,51 \%$ frente a una media de 7,26\%). Asimismo, destacan con respecto a la media Europa Occidental y Norteamérica como principales destinos, figurando también Europa del Este como destino significativamente más frecuente que para el conjunto de la población (4,68\% de las estancias frente a una media de 3,34\%). América Latina tiene un peso significativamente bajo en contraste con otras disciplinas.

- TEP: Tecnologías de la Producción (339 investigadores)

La movilidad en esta área se caracteriza por un número de estancias por investigador $(2,19)$ inferior al resto de las áreas y, por tanto, por un peso significativamente mayor de los investigadores que declaran una única estancia. Destaca por encima de las demás áreas Norteamérica como destino importante. 
- BIO: Biología (440 investigadores)

La movilidad en este grupo es también significativamente inferior a la media (2,32 estancias por investigador). Destacan en él las estancias de mayor duración; $16,78 \%$ de las estancias tienen una duración de 12 a 24 meses frente a un promedio de 7,26\%. Se trata del grupo con mayor peso de las estancias de mayor duración. Europa Occidental y Norteamérica son destinos significativamente más frecuentes que para el conjunto de las áreas, al contrario que América Latina y África. Las estancias totales de este grupo están significativamente más concentradas en las décadas de 1980 y 1990 y menos entre 2000 y 2010.

- RNM: Recursos Naturales y Medio Ambiente (590 investigadores)

Las únicas pautas diferenciales de esta área de conocimiento son su ligera mayor movilidad por investigador con respecto a la media (2,98 estancias por investigador frente a una media de 2,78) y el peso significativamente superior de las estancias de larga duración, aunque sin llegar a alcanzar los niveles de Biología.

- CTS: Ciencia y Tecnología de la Salud (443 investigadores)

Este grupo presenta una movilidad significativamente inferior a los demás en conjunto, en términos de estancias por investigador (2,19 estancias por investigador), aunque destaca por haber sido más móvil que la media en los períodos anteriores a 2000. Los investigadores de este grupo van significativamente más a Norteamérica y realizan más estancias de larga duración que la media (12,4\% de las estancias con duración de 12 a 24 meses).

- TIC: Tecnologías de la Comunicación (412 investigadores)

Las pautas en este grupo son similares a las del anterior (CTS): movilidad por investigador inferior a la media (2,26 estancias por investigador) y preferencia por Norteamérica como destino en comparación con las demás (cabe recordar que Europa occidental se mantiene como destino más frecuente para todas las áreas). El grupo se distingue de CTS, en cambio, por registrar una movilidad significativamente mayor en la última década y menor en períodos anteriores.

- AGR: Agricultura (396 investigadores)

Finalmente, este grupo también destaca por una movilidad menos frecuente que otros y de mayor duración, así como por una disminución en su peso en la última década en comparación con las dos anteriores.

\section{Conclusiones: discusión de resultados}

Los resultados alcanzados en este estudio tienen una doble relevancia. Por una parte constituyen una aportación metodológica, al poner de manifiesto el 
potencial de los sistemas de información curricular y de sus bases de datos electrónicas para el análisis de la movilidad de los investigadores. Las políticas públicas de investigación, tanto a nivel regional, como nacional, como europeo, vienen asignando una importancia y una cantidad de fondos crecientes al fomento de la movilidad de los investigadores. No obstante, las características del fenómeno siguen siendo en gran medida desconocidas y las fuentes estadísticas tradicionales disponibles (encuestas de I+D, de migraciones, censos, estadísticas de educación, etc.) no resultan útiles para estudiarlo. Los estudios empíricos realizados hasta la fecha, tanto en nuestro país como en otros, han estado basados en el análisis de muestras de reducido tamaño en la mayoría de los casos y de escasa representatividad. No obstante, encontramos relevantes consistencias entre nuestros resultados y los de otros estudios realizados en España sobre otras poblaciones, especialmente en cuanto a la distribución por género de las estancias, su destino, su frecuencia y su duración.

La contribución fundamental de este trabajo, desde el punto de vista metodológico, es que analiza la totalidad de la población objeto de estudio, es decir, la población de investigadores andaluces que han declarado alguna estancia de investigación en el extranjero, de una semana a dos años de duración, en sus currículos. Se trata de un total de 6.345 investigadores cuyos datos socio demográficos y de movilidad han sido directamente descargados del Sistema de Información Científica de Andalucía (bajo estricto control de la confidencialidad de los datos identificadores de cada individuo, como nombres o apellidos).

El estudio pone, por tanto, de manifiesto el potencial de sistema nacional de información curricular que se encuentra en desarrollo en el conjunto de España, gracias al estándar de comunicación CVN-XML desarrollado por la Fundación Española para la Ciencia y la Tecnología. La interconexión de las bases de datos curriculares disponibles en el país permitirá, en el futuro próximo, el análisis de poblaciones más numerosas que la aquí estudiada.

La indiscutible representatividad de los datos empleados hace, por otra parte, que los resultados sean relevantes en sí mismos, al poner de manifiesto una serie de tendencias relativas a la movilidad temporal analizada, de duración comprendida entre una semana y dos años.

Cabe resaltar, en primer lugar, que la población de investigadores cuyo currículo registra alguna estancia en el extranjero de las características apuntadas representa el 22\% del total de investigadores andaluces, porcentaje que no es directamente comparable con el de otros estudios disponibles pero que no parece ser significativamente distinto al de éstos. No deja de ser relevante el hecho de que las tres cuartas partes de los investigadores no hayan realizado ninguna estancia internacional. La tendencia registrada a lo largo del tiempo pone de manifiesto, sin embargo, un crecimiento sostenido en el número de estancias por investigador desde mediados de la década de 1980 hasta la actualidad, alcanzando un nivel máximo medio, para la población móvil (la que ha declarado al menos una estancia en el currículo), de una estancia cada 4 años y de una duración media comprendida entre 1 semana y 3 meses. 
Además de la posibilidad que brindan los datos de cuantificar la movilidad y observar su evolución, los resultados desvelan tendencias interesantes, relativas a lo que podríamos llamar "relevos" de diverso orden: relevo de género, relevo disciplinario y relevo geográfico. El relevo de género hace referencia a la progresiva disminución de la distancia entre hombres y mujeres registrada, a medida que nos desplazamos hacia cohortes más jóvenes de población. En éstas se observa una mayor presencia de mujeres en la población (especialmente en las áreas de biología, ciencia y tecnología de la salud, humanidades y recursos naturales y medioambiente) y una mayor frecuencia en la movilidad, hasta el punto en que la significatividad estadística en las diferencias por género desaparece.

El relevo disciplinario viene marcado por la creciente participación en la movilidad temporal de áreas de conocimiento con menor tradición de movilidad, como las humanidades o las ciencias sociales, que aquéllas en las cuales la realización de estancias internacionales ha estado estrechamente ligada a la promoción profesional desde hace tiempo, como la biología, la física, química y matemáticas o las ciencias y tecnologías de la salud. En estas últimas áreas, las realización de estudios postdoctorales en el extranjero de duración comprendida entre 12 y 24 meses, es una práctica más habitual, mientras que no lo es en el caso de las humanidades y las ciencias sociales. La movilidad temporal registrada en estas disciplinas tiene otras características: es más frecuente y de duración más corta. El estudio europeo anteriormente citado, relativo a los obstáculos a la movilidad de los investigadores en Europa (CEC, 2008b) y basado en la realización de encuestas, señala que el número de investigadores móviles (residentes en un país distinto al de origen en el momento de contestar la encuesta) es superior en las ciencias de la vida que en las humanidades y ciencias sociales. A la vista de nuestros resultados, podemos apuntar que estas conclusiones pueden estar sesgadas por la definición de "investigador móvil" que guía el citado estudio europeo.

Al ser la movilidad de los investigadores de ciencias humanas y sociales aparentemente más frecuente y más corta, será más difícil encontrarlos residiendo en un país distinto al de origen, lo que no implica que sus movimientos no desempeñen un papel fundamental en la evolución y transmisión de conocimiento y en la integración de equipos transnacionales en estas disciplinas. Nuestro estudio confirma que los perfiles de movilidad difieren notablemente entre áreas de conocimiento y que existen culturas específicas de movilidad en función de las disciplinas (Ackers, 2008), lo cual no puede ser ignorado por las políticas públicas, ni a la hora de diseñar instrumentos de apoyo a la movilidad, ni a la hora de evaluar la actividad de los investigadores. La "expectativa de movilidad" que se está convirtiendo crecientemente en un elemento de evaluación de la evolución de las carreras investigadoras (Ackers, 2008), debería ajustarse a estas diferentes prácticas y culturas.

Finalmente, el relevo geográfico está directamente ligado al relevo disciplinario. La creciente realización de estancias internacionales por parte de los inves- 
tigadores de ciencias sociales y humanas está contribuyendo a la emergencia de nuevos destinos geográficos para la movilidad a países cuyos centros de investigación están ganando atractivo en el resto del mundo, especialmente en áreas en las que la investigación no requiere de grandes inversiones en capital tecnológico y material de laboratorio. Es llamativo el crecimiento de las estancias en América Latina a lo largo del período 2000-2010 y la creciente participación de países africanos como destino, notablemente Marruecos, cuya cercanía geográfica a Andalucía podría explicar esta tendencia. Los destinos de la mayoría de las estancias siguen siendo Europa Occidental y del Norte, y Estados Unidos sigue atrayendo a un buen número de investigadores, especialmente de las áreas de ciencias puras, de la vida y de la salud. Lo anterior no disminuye, sin embargo, la relevancia de la emergencia de nuevos destinos, lo cual contribuye a reconfigurar los espacios internacionales de investigación y colaboración. En el caso de Andalucía, se hace especialmente patente la creciente conexión con América Latina.

Los resultados obtenidos a partir de los datos disponibles para realizar este trabajo ponen de relieve realidades y tendencias potencialmente relevantes para el diseño de políticas de investigación, pero no son sino un primer paso en el estudio de las dinámicas de movilidad basado en datos electrónicos curriculares. No hemos explotado aquí el potencial de información longitudinal que ofrece el currículo. Sería interesante ver en qué momento de la carrera del investigador se produce la movilidad, ya sea ésta temporal y geográfica o de otro tipo, y los efectos de la misma en términos de producción científica, establecimiento de redes de colaboración y co-producción, promoción profesional, etc. Sería asimismo recomendable comparar los perfiles de la población móvil con los de la población, mucho más numerosa, que no realiza estancias, con objeto de valorar los efectos tanto de la movilidad como de la inmovilidad. Las limitaciones de espacio y de posibilidades de análisis derivadas de los datos descargados del sistema de información hasta la fecha, nos llevan a dejar abiertas estas opciones de análisis para futuras investigaciones.

\section{Agradecimientos}

Los autores desean agradecer a Samaly Santa, de la Consejería de Innovación, Ciencia y Empresa de la Junta de Andalucía, su inestimable ayuda en la descarga de los datos utilizados. Asimismo, agradecen a Florencio Núñez, de la Fundación Española para la Ciencia y la Tecnología su ayuda para la actualización de la información referente al proyecto Curriculum Vitae Normalizado. El estudio ha sido realizado en el marco de las actividades del proyecto EURO-CV, de la Red de Excelencia Europea PRIME (CIT1-CT-2003-506596), financiada por el Sexto Programa Marco de la Unión Europea y del proyecto CV-IBEROAMERICANO (A/9107/07), financiado por la Agencia Española de Cooperación Internacional y Desarrollo.

Rev. Esp. Doc. Cient., 33, 3, julio-septiembre, 428-457, 2010. ISSN: 0210-0614. doi:10.3989/redc.2010.3.736 


\section{Bibliografia}

Ackers, L. (2005). Moving people and knowledge: Scientific mobility in the European Union. International Migration, vol. 43 (5), 99-131.

Ackers, L. (2008). Internationalisation, mobility and metrics: a new form of indirect discrimination? Minerva, vol. 46, 411-435

Báez, J. M.; Peset, F.; Núñez, F., y Ferrer, A. (2008). CVN: La normalización de los currículos científicos. El Profesional de la Información, vol. 17 (2), 213-220.

Barjak, F., y Robinson, S. (2008). International collaboration, mobility and team diversity in the life sciences: impact on research performance. Social Geography, vol. 3, 23-36.

Bhagwati, J. N. (1979). International Migration of the Highly Skilled: Economics, Ethics and Taxes. Third World Quarterly, vol. 1 (3), 17-30.

Bozeman, B.; Dietz, J., y Gaughan, M. (2001). Scientific and Technical Human Capital: An Alternative Model for Research Evaluation International Journal of Technology Management, vol. 22 (7/8), 716-740.

Bozeman, B. (2008). Capital Humano Científico y Técnico: evaluación de la investigación desde una aproximación basada en las capacidades. En Cañibano, C.; Encinar, M. I., y Muñoz, F. (eds.), Economía del Conocimiento y la Innovación: Nuevas aproximaciones a una relación compleja. Ediciones Pirámide, Madrid, 59-86.

Brandi, M. C. (2006). The historical evolution of highly qualified migrations. http://www. irpps.cnr.it/sito/curricula/curr_brandi.htm, Versión original en italiano: Studi Emigrazione. Vol. XLI, $\mathrm{n}^{\circ}$ 156, diciembre de 2004.

Cañibano, C.; Bozeman, B. (2009). Curriculum Vitae Method in Science Policy and Research Evaluation: The State-of-the-Art. Research Evaluation, vol. 18 (2), 86-94.

Cañibano, C. (2008). Movilidad de investigadores y flujos de conocimiento: hacia una revisión necesaria de la teoría del capital humano. En Cañibano, C.; Encinar, M. I., y Muñoz, F. (eds.), Economia del Conocimiento y la Innovación: nuevas aproximaciones a una relación compleja. Pirámide, Madrid, 87-111.

Cañibano, C.; Otamendi, J., y Andújar, I. (2008). Measuring and assessing researcher mobility from CV analysis: the case of the Ramon y Cajal programme in Spain. Research Evaluation, vol. 17 (1), 17- 31.

CEC. A Mobility Strategy for the European Research Area. Communication from the Commission to the Council and the European Parliament. Brussels, 2001, COM (2001) 331 final.

CEC. The European Research Area: New perspectives. Green Paper. Brussels, 2007, COM (2007) 161 final.

CEC. Realising a single labour market for researchers. Report of the ERA expert group. 2008a EUR23321EN.

CEC. Evidence on the main factors inhibiting mobility and career development of researchers; by IDEA Consult (coord.), Fraunhover - ISI, NIFU STEP, PREST, SPRU and Technopolis. 2008b, Contract DG-RTD-2005-M-02-01.

Consejo Europeo de Lisboa. Conclusiones de la Presidencia. 23 y 24 de marzo de 2000. Nr: 100/1/00. http://ue.eu.int/Newsroom/LoadDoc.asp? BID=76\&DID=60932\& LANG=1.

Rev. Esp. Doc. Cient., 33, 3, julio-septiembre, 428-457, 2010. ISSN: 0210-0614. doi:10.3989/redc.2010.3.736 
Corley, E., Bozeman, B., y Gaughan, M. (2003). Evaluating the impacts of grants on women scientists' careers: the curriculum vitae as a tool for research assessment. En Shapira, P., y Kuhlmann, S. (eds.), Learning from Science and Technology Policy Evaluation: Experiences from the U.S. and Europe. Cheltenham, UK: Edward Elgar Publishing, 293-315.

De Filippo, D. (2008). Movilidad y producción científica en la UC3M. Estudio de la actividad científica del profesorado a partir de bases de datos institucionales (Universitas XXI) y bibliográficas (WoS, ICYT, ISOC) (1997-2005). [Tesis doctoral] Universidad Carlos III de Madrid.

De Filippo, D.; Sanz Casado, E., y Gómez, I. (2007). Movilidad de investigadores y producción en coautoría para el estudio de la colaboración científica. CTS, Cienc. Tecnol. Soc. vol. 3 (8), 23-40.

De Filippo, D.; Sanz Casado, E., y Gómez, I. (2009). Quantitative and qualitative approaches to the study of mobility and scientific performance: a case study of a Spanish university. Research Evaluation, vol. 18 (3), 191-200.

Dietz, J.; Chompolov, I.; Bozeman, B.; Lane, E., y Park, J. (2000). Using the curriculum vitae to study the career paths of scientists and engineers: An exploratory assessment. Scientometrics, vol. 49 (3), 419-442

Dietz, J., y Bozeman, B. (2005). Academic careers, patents and productivity: industry experience as scientific and technical human capital. Research Policy, vol. 34, 349-367.

D'Onofrio, M.G. (2009). The Public CV Database of Argentine Researchers and the "CVminimum". Latin-American Model of Standardization of CV Information for R\&D Evaluation and Policy Making. Research Evaluation, vol. 18 (2), 95-103.

Ekeland, A. (2008). The emerging electronic CV systems in Norway. National Report from Norway to the PRIME NoE project EURO-CV.

Fontes, M. (2007). Scientific Mobility Policies: How Portuguese Scientists envisage the return home. Science and Public Policy, vol. 34 (4), 284-298

Fontes, M. (2008). National Report from Portugal to the PRIME NoE project EURO-CV.

Gaillard, A. M., y Gaillard, J. (1997). The international mobility of brains: exodus or circulation? Science Technology \& Society, vol. 2, 195-228

García Romero, A. (2000). El efecto de la estancia postdoctoral en la productividad científica. Universidad Carlos III, Documento de Trabajo 00-01.

Gaughan, M., y Bozeman, B. (2002). Using curriculum vitae to compare some impacts of NSF research center grants with research center funding. Research Evaluation, vol. 11 (1), 17-26.

Gaughan, M., y Robin, S. (2004). National science training policy and early scientific careers in France and the United States. Research Policy, vol. 33, 569-581

Johnson, H. G. (1968). An "Internationalist model". En Adams, W. (ed.), The Brain Drain, Chapter 5, 69-91.

Jonkers, K., y Tijssen, R. (2008). Chinese researchers returning home: impacts of international mobility on research collaboration and scientific productivity, Scientometrics, vol. 77 (2), 309-333.

Kapur, D., y McHale, D. (2005). Give me your best and your brightest. Center for Global Development, Washington D.C. 
Lee, S., y Bozeman, B. (2005). The Impact of Research Collaboration on Scientific Productivity. Social Studies of Science, vol. 35 (5), 673-702.

Martin-Sempere, M. J.; Plaza, L. M., y Rey Rocha, J. (1997). La movilidad temporal de los investigadores postdoctorales en el extranjero. Política Científica, vol. 47, 50-53.

Martín-Sempere, M. J.; Rey, J., y Plaza, L. M. (1999). Movilidad temporal de investigadores y cooperación científica internacional. Las estancias de los sabáticos latinoamericanos en España. Interciencia, vol. 4 (2), 107-111

Martín-Sempere M. J., y Rey Rocha, J. (2003). Evaluacion y seguimiento de programas de movilidad del personal investigador. CSIC. Grupo de Estudios de la Actividad Científica. Informe EA2003-0028. http://digital.csic.es/handle/10261/2996.

Meyer, J. B. (2001). Network approaches versus brain drain lessons from the diaspora. International Migration, vol. 39 (5) Special Issue 1, 99-110.

Meyer, J. B.; Kaplan, D., y Charum, J. (2001). Scientific nomadism and the new geopolitics of knowledge, International Social Science Journal, vol. 53 (168), 309-321.

OCDE. Frascati Manual 2002: proposed standard practice for surveys on research and experimental development. París, 2002.

OCDE. The Global Competition for Talent. Mobility of the Highly Skilled. París, 2008.

Sabatier, M.; Carrere, M., y Mangematin, V. (2006). Profiles of Academic Activities and Careers: DoesGender Matter? An Analysis Based on French Life Scientists' CVs. Journal of Technology Transfer, vol. 31, 311-324.

Sandström, U. (2009). Combinig curriculum vitae and bibliometric analysis: mobility, gender and research performance. Research Evaluation, vol. 18 (2), 135-142.

Saxenian, A. L. (2006). The New Argonauts: regional advantage in a global economy. Cambridge, MA. Harvard University Press.

Shinn, T., y Benguigui, G. (1997). Physicist and intellectual mobility. Social Science Information, vol. 36 (2), 293-309.

Solís Cabrera, F. M. (2008). El Sistema de Información Científica de Andalucía, una experiencia pionera en España. Las Comunidades Autónomas frente a la $I+D+i$. Madri+d diciembre, $12-18$.

US House of Representatives Committee on International Relations Brain Drain: A Study of the Persistent Issue of International Scientific Mobility, Ch.13 in Science, Technology, and American Diplomacy, 3 Volumes, US Government Printing Office, Washington, 1977, 1037-1318.

Woolley, R., y Turpin, T. (2009). CV analysis as a complementary methodological approach: Investigating the mobility of Australian scientists. Research Evaluation, vol. 18 (2), 143-151.

Zubieta, A. (2009). Acknowledgement of weak ties: Is there a positive effect of postdoctoral position on academic performance and career development? Research Evaluation, vol. 18 (2), 105-115. 